

\title{
Marché du travail, évaluation et économie expérimentale
}

Nicolas Jacquemet, Olivier L'haridon, Isabelle Vialle

\section{To cite this version:}

Nicolas Jacquemet, Olivier L'haridon, Isabelle Vialle. Marché du travail, évaluation et économie expérimentale. Revue Française d'Economie, 2014, XXIX (1), pp.189-226. 10.3917/rfe.141.0189 . halshs-01082352

\section{HAL Id: halshs-01082352 \\ https://shs.hal.science/halshs-01082352}

Submitted on 13 Nov 2014

HAL is a multi-disciplinary open access archive for the deposit and dissemination of scientific research documents, whether they are published or not. The documents may come from teaching and research institutions in France or abroad, or from public or private research centers.
L'archive ouverte pluridisciplinaire HAL, est destinée au dépôt et à la diffusion de documents scientifiques de niveau recherche, publiés ou non, émanant des établissements d'enseignement et de recherche français ou étrangers, des laboratoires publics ou privés. 


\section{Marché du travail, évaluation et économie expérimentale}

Nicolas Jacquemet ${ }^{1}$
Olivier l'Haridon ${ }^{2}$

Isabelle Vialle ${ }^{2}$

Mars 2014

\section{Résumé}

L'économie comportementale et l'économie expérimentale ont connu un formidable essor au cours des dernières décennies. L'objectif de cet article est de présenter, au travers d'un cadre général d'analyse et d'exemples d'applications, l'intérêt de l'économie expérimentale pour l'évaluation des politiques publiques sur le marché du travail. L'économie expérimentale et les méthodes alternatives existantes apparaissent comme des outils d'analyse complémentaires dans l'étude des politiques publiques. En particulier, les résultats issus de l'économie expérimentale apportent un éclairage critique sur les modèles théoriques sousjacents à l'étude des politiques du marché du travail.

\footnotetext{
${ }^{1}$ Université de Lorraine, BETA et Ecole d'Economie de Paris.

${ }^{2}$ Université de Rennes 1, CREM et TEPP.

Nous remercions deux rapporteurs anonymes, Laurent Denant-Boëmont, François Langot, Yannick L'Horty, Stéphane Robin, Philippe Zamora et l'ensemble des participants à l'école thématique TEPP pour leurs remarques et leurs suggestions.
} 


\section{Introduction}

La méthode expérimentale a été pendant longtemps l'instrument d'observation empirique privilégié des travaux d'économie comportementale. Cette méthode consiste à créer un environnement contrôlé afin de (re)créer artificiellement une situation reflétant les conditions d'un comportement économique. Comme toute méthode expérimentale, elle se fonde sur la formulation d'hypothèses, l'observation empirique par l'expérience et l'interprétation des résultats. La méthode expérimentale est maintenant devenue un élément à part entière de la boîte à outils de l'économiste. A ce titre elle peut être mobilisée pour évaluer des politiques publiques, notamment concernant le marché du travail. L'avantage premier de l'économie expérimentale repose sur ses capacités de contrôle de l'observation empirique : contrôle de l'assignation aléatoire des participants, contrôle de la nature des institutions, contrôle de la spécification des variables exogènes, contrôle des processus de détermination des variables endogènes.

Parallèlement à cet avantage indéniable qui la distingue des autres méthodes empiriques, l'économie expérimentale est depuis longtemps reconnue pour servir trois objectifs (Roth [1988] ; [2010]). Le premier consiste à tester la théorie économique en laboratoire : tester certaines de ses hypothèses (égoïsme de l'agent économique, autonomie de la volonté, optimalité des décisions prises par les individus), tester ses prédictions notamment pour des objets habituellement non - ou difficilement - observables (salaires de réservation, croyances, anticipations), fournir des mesures comportementales (aversion au risque, impatience, aversion à l'inégalité). Le deuxième consiste à faire émerger des faits et des comportements qui ne sont pas - ou peu - envisagés habituellement par la théorie économique (sensibilité aux iniquités, normes sociales et phénomènes de groupe, confiance) et à étendre ainsi son champ d'application. Le dernier objectif est de murmurer à l'oreille des princes et participer ainsi à l'élaboration et à l'évaluation des politiques publiques.

Cet article se concentre plus particulièrement sur le premier et sur le dernier rôle de l'économie expérimentale, avec un double objectif. Le premier est de montrer l'intérêt des méthodes de l'économie expérimentale pour l'évaluation des politiques publiques, en regard notamment de leur parenté et de leur complémentarité avec les méthodes existantes (estimations ex post, expériences naturelles, évaluations ex ante par assignation aléatoire par exemple). Le second objectif est d'apporter un éclairage critique sur les modèles 
théoriques sous-jacents à l'étude des politiques du marché du travail, à l'aide des résultats issus de l'économie expérimentale.

Dans le champ de l'économie du travail, l'économie expérimentale a connu un formidable essor au cours des 15 dernières années et s'est appliquée à cinq domaines principaux (Charness et Kuhn [2011]). Le premier concerne les interactions principal-agent, en particulier les modes de rémunération et l'offre d'effort. Les résultats montrent l'importance des effets de pairs dans la performance individuelle et la réponse aux incitations (Gneezy et Rustichini [2000] ; Falk et Ichino [2006] ; Bandiera et al. [2010]). Plus généralement, les préférences dites «sociales », qui font dépendre les décisions d'un individu de la situation des autres, ont d'ailleurs été l'objet majeur des articles d'économie expérimentale publiés au cours des dix dernières années, soulignant le rôle potentiellement important que peut jouer cette composante des préférences dans les comportements individuels. Le deuxième domaine concerne les négociations et les conflits de travail. Les résultats montrent que les comportements observés en laboratoire divergent fortement des prédictions théoriques, même dans les cas les plus simples comme le jeu du dictateur ou le jeu de l'ultimatum ${ }^{1}$. Le troisième domaine concerne les différences de comportement liées aux caractéristiques des individus (sexe, âge, origine, apparence) et les questions traditionnelles de discrimination qui y sont attachées (Fershtman et Gneezy [2001] ; Croson et Gneezy [2009] ; Niederle et Vesterlund [2007]). Les deux derniers domaines, qui seront plus précisément développés ici, concernent l'étude de l'équilibre du marché du travail (mécanismes d'appariement, aléa moral, sélection adverse) et les comportements de prospection d'emploi.

Cet article se compose de trois parties. Dans la première, nous présentons la méthode expérimentale en économie en insistant plus particulièrement sur ses avantages et ses limites dans une perspective d'évaluation des politiques publiques. Ces principes généraux sont ensuite illustrés à travers deux exemples d'applications. Nous présentons d'abord les travaux d'évaluation consacrés aux marchés d'appariement. Nous nous penchons ensuite sur l'approche expérimentale des modèles de prospection d'emploi et sur la pertinence de leurs hypothèses comportementales dans une perspective d'évaluation ex ante des politiques du marché du travail.

\footnotetext{
${ }^{1}$ Dans les deux cas, deux parties disposent d'une dotation initiale et l'une d'entre elles doit proposer un partage de cette dotation. Dans le jeu du dictateur, l'autre partie ne peut pas rejeter l'offre, dans le jeu de l'ultimatum le partage proposé n'est effectif que si l'autre partie l'accepte (sinon la dotation tombe à zéro).
} 


\section{Principes et méthodes de l'économie expérimentale}

L'utilité de l'économie expérimentale à des fins d'évaluation des politiques publiques tient à la position particulière qu'elle occupe, à l'intersection entre la modélisation théorique et l'observation empirique. De ce point de vue, sa spécificité est d'offrir un contrôle très fort de l'environnement des décisions, mais au prix d'une perte de réalisme qui rend parfois plus délicate que pour d'autres méthodes empiriques la généralisation des résultats observés aux circonstances économiques d'intérêt.

\subsection{Réalités économiques, théorie et expériences en laboratoire}

Comme le montrent les trois objectifs fondateurs rappelés en introduction, l'économie expérimentale se situe d'un point de vue méthodologique à l'intersection entre la théorie économique, qu'elle cherche à tester et à opérationnaliser, et la réalité que toutes deux s'efforcent d'éclairer.

Pour comprendre les conditions dans lesquelles ces trois objets d'analyse dialoguent entre eux, Samuelson [2005] propose une formalisation fondée sur deux types d'ensemble : les inputs, notés $X$, qui désignent l'ensemble des éléments causaux en présence; et les outputs ou conséquences, notés $S$, qui correspondent à l'ensemble des phénomènes engendrés par les premiers.

A partir de cette typologie, la réalité peut être représentée comme une transformation de la combinaison infinie de causes $X^{\infty}$ vers une combinaison infinie de conséquences $S^{\infty}$ :

$$
F: X^{\infty} \rightarrow S^{\infty}
$$

Si cette "définition " est aussi sommaire que simpliste, elle a le mérite de mettre en évidence la raison première de la nécessité de la production de connaissances scientifiques (en sciences sociales en l'occurrence) pour comprendre les mécanismes à l'œuvre dans la vie (économique) : il s'agit de rendre intelligible un environnement dont la complexité dépasse irrémédiablement les capacités de l'esprit humain, en raison tant de la combinaison infinie des mécanismes impliqués que de l'infinité des dimensions pertinentes.

En économie, la modélisation théorique est le mode privilégié de production de connaissances. Dans les termes décrits plus haut, la modélisation théorique opère d'abord une réduction de la réalité étudiée, à travers le choix d'un sous-ensemble d'inputs de dimension finie, $N$. Elle opère ensuite une réduction du prisme à travers lequel cette réalité est comprise, par l'intermédiaire du choix d'un sous-ensemble fini de dimension $M$ des 
conséquences que la modélisation vise à capturer. Enfin, la modélisation théorique consiste à produire une relation causale entre ces deux sous-ensembles : 2

$$
f: X^{N} \rightarrow S^{M}
$$

Ce processus de réduction du phénomène étudié à un petit nombre de causes et de conséquences d'intérêt est le principal point commun entre la modélisation théorique et la construction d'expériences en laboratoire. La différence essentielle tient au fait que les expériences consistent à construire des situations réelles, plutôt que des représentations intellectuelles : dans une expérience, des individus prennent des décisions et réagissent à l'environnement. Aux $n$ inputs sur lesquels l'expérience se concentre, notés $x^{n}$ et dont la valeur est contrôlée par l'expérimentateur par l'intermédiaire du protocole de l'expérience, s'ajoute ainsi une infinité d'éléments qui échappent à ce choix, tels que l'histoire individuelle des participants, le degré de confort avec lequel ils sont installés ou encore les dispositions psychologiques dans lesquelles ils se présentent au laboratoire. L'ensemble de ces éléments est susceptible d'influencer les décisions mais échappe au contrôle de l'expérimentateur comme à l'observation, de sorte que les déterminants des décisions effectivement en présence sont $x^{\infty}=x^{n} U x^{\infty-n}$.

La seconde réduction opérée lors de la construction de l'expérience est le choix de mesures, en nombre $m$, qui correspondent aux conséquences de l'environnement qui seront effectivement observées et enregistrées. II s'agit notamment des décisions prises par les participants, de leurs interactions avec d'autres et de l'historique de leurs choix, mais aussi par exemple des temps de décision ou encore des réactions sensorielles mesurées par des capteurs physiologiques, ou des changements psychologiques mesurés à l'aide d'enquêtes adjointes à l'expérience. Ces mesures relèvent du choix de l'expérimentateur au même titre que les inputs : les variations de température à l'intérieur du laboratoire en raison de l'activité des participants, par exemple, font partie des conséquences effectives de l'environnement qui resteront non mesurées dès lors que leur mesure n'est pas directement incluse dans le protocole.

Sur cette base, les expériences en laboratoire produisent donc des observations qui s'écrivent : $F^{m}\left(x^{\infty}\right)$. Ces observations correspondent à la fonction de réaction empirique des participants de l'expérience : elles renseignent sur les décisions effectivement induites par

\footnotetext{
${ }^{2}$ Dans l'article original de Samuelson [2005], ces définitions sont développées en termes de distribution de probabilités sur l'ensemble de conséquences. Cette généralisation permet de prendre en compte le fait que la modélisation théorique n'a pas vocation à expliquer le phénomène d'intérêt de façon strictement déterministe, mais bien à isoler les forces à l'œuvre. Nous ignorons cet aspect ici par souci de simplification.
} 
l'environnement étudié par la modélisation théorique. En ce sens, la méthode expérimentale fournit un contre factuel empirique aux prédictions de l'analyse théorique.

\subsection{Les avantages de l'approche expérimentale}

L'économie expérimentale se distingue des autres méthodes empiriques mobilisées pour l'analyse économique avant tout par les capacités de contrôle qu'elle offre sur l'environnement des décisions. Le contrôle s'opère tout d'abord sur le biais de sélection qui est susceptible d'affecter la mesure de l'effet causal d'intérêt : les participants sont assignés aléatoirement à différents groupes qui constituent autant de groupes de traitement car chaque groupe fait face à une situation propre ${ }^{3}$. La capacité de contrôle des conditions expérimentales s'applique ensuite au contrôle des variables exogènes. La grande force des expériences de laboratoire est qu'elles permettent de mettre en place des variations ceteris paribus, qui autorisent ainsi des observations empiriques directes de statique comparative, comparables à celles qui sont présentées dans les manuels d'analyse économique. Un exemple notable est la variation de l'information dont disposent les participants, qui permet d'atteindre une finesse et une précision d'observation difficilement atteignables par les autres méthodes empiriques. Ceci explique l'importance des résultats expérimentaux dans la littérature en théorie des jeux et en théorie de la décision par exemple. Dans ces domaines, la méthode expérimentale autorise des variations extrêmement précises de l'information. Ces variations peuvent consister à spécifier de manière fine l'information dont dispose chaque joueur dans un jeu et permettent par exemple d'étudier précisément l'information asymétrique dans les jeux (Roth [1995]). La variation de l'information peut également porter sur la quantité d'information dont disposent les individus en environnement incertain (Ellsberg [1961] ; Hertwig et al. [2004]). Ces variations rendent cette méthode indispensable aux progrès théoriques et au développement de nouveaux modèles (Camerer [2003] ; Machina [2009]; Wakker [2010]). Dans la pratique, les conséquences réelles pour les participants de leurs actes (ce que l'économie expérimentale appelle couramment les «incitations ») et les règles de l'expérience sont les principaux instruments de contrôle sur lesquels agit l'expérimentateur. Les possibilités de contrôle ne se limitent pas à l'environnement de choix ou aux conditions informationnelles, elles autorisent également des

\footnotetext{
${ }^{3}$ II convient ici de noter que le contrôle du biais de sélection ne s'applique qu'à l'assignation à un "traitement" et non à la participation : le choix de participation à une expérience reste ainsi soumis aux biais habituels dans ce domaine. Si cette limite reste peu importante lorsque les participants ne connaissent pas à l'avance le sujet et les conditions d'une expérience, la situation est susceptible d'être différente si l'information sur les conditions de l'expérience est diffusée à l'extérieur du laboratoire ou si les participants doivent s'engager à participer à des sessions expérimentales répétées.
} 
mesures psychologiques réalisées sous forme de tests déclaratifs (Anderson et al. [2011]) ou physiologiques (taux de pression sanguine, taux de testostérone, imagerie cérébrale, mesures transcutanées) associées aux décisions économiques. Comme le soulignent Falk et Heckman [2009], l'ensemble de ces capacités de contrôle permet à l'économiste d'obtenir des observations empiriques "sur-mesure », lui assurant de ce fait des tests directs de ses modèles.

Les méthodes expérimentales présentent l'avantage de pouvoir tester de manière explicite des prédictions théoriques de l'analyse économique. Cette capacité prédictive a été largement mobilisée pour les décisions individuelles (Kahneman et Tversky [1979]), la théorie des jeux (Roth [1995] ; Camerer [2003]), les décisions intertemporelles (Coller et Williams [1999]), les équilibres de marché (Smith [1962]), les biens publics (Davis et Holt [1993] ; Ledyard [1994]). L'observation peut en effet se porter sur de nombreux objets difficilement observables en dehors du laboratoire : conditions d'équilibre (existence, unicité, stabilité), dynamiques d'ajustement, propriétés hors de l'équilibre, stratégies individuelles, règles optimales. L'avantage des méthodes expérimentales réside ici dans leur capacité à dessiner une véritable carte des conditions dans lesquelles les comportements réels correspondent aux prédictions théoriques. Les mesures réalisées permettent ainsi d'apprécier quantitativement l'écart séparant les comportements observés et les comportements prédits par un modèle théorique. Dans une perspective de modélisation « as if » à la Friedman et Savage [1948] $]^{4}$, le modèle n'est donc pas remis en question sur la base de l'irréalisme de ses hypothèses comportementales, mais bien sur celui de ses prédictions. L'objectif empirique n'est donc pas la simple réfutation d'une théorie mais au contraire d'identifier les directions dans lesquelles elle doit progresser pour augmenter son pouvoir de prédiction. C'est le cas par exemple lorsque les théories standard du choix dans l'incertain sont insuffisantes pour décrire les comportements observés et doivent être complétées par des théories prenant en compte les doutes de l'agent économique par exemple.

L'avantage des méthodes expérimentales en économie ne se limite pas à leur capacité de contrôle ou à leur capacité prédictive. Un troisième avantage est lié à la possibilité qu'elles offrent de répliquer les résultats de manière presque illimitée (Falk et Heckman [2009]). Ainsi la significativité empirique des résultats expérimentaux est assurée, moins par la taille de l'échantillon ou la nature de la population étudiée que par la régularité

\footnotetext{
${ }^{4}$ Le "as if" correspond à un argument pour défendre l'irréalisme des hypothèses d'un modèle d'analyse économique. Selon cet argument, un modèle peut se contenter de représenter les comportements individuels « comme si » les agents étaient pleinement rationnels, à partir du moment où ses conclusions reproduisent correctement les comportements réels observés sur les marchés.
} 
des phénomènes observés sur de nombreuses réplications. Le croisement du contrôle et des possibilités de réplication permet d'étudier des variations à la marge des conditions expérimentales et de tester ainsi la robustesse des résultats obtenus. Les résultats relatifs au jeu de l'ultimatum, fondement de l'approche stratégique de la négociation à la Nash (Rubinstein [1982]), ont ainsi été testés dans une multitude de configurations (Roth [1995]) 5 qui permettent d'en affiner les conclusions. La capacité de réplication des méthodes expérimentales présente un intérêt particulier lorsqu'elle est envisagée en complémentarité avec les autres méthodes d'observation empiriques. Par exemple, les méthodes issues de l'économie expérimentale peuvent être intégrées dans des enquêtes, des panels d'interviewés (Dohmen et al. [2011]) ou encore des expériences de terrain (List et Rasul [2011] ; Bandiera et al. [2010]), afin d'évaluer la sensibilité des résultats à des situations alternatives au laboratoire. Enfin, la réplication permet de comparer les réactions des étudiants (qui constituent la population habituellement mobilisée dans les expériences en laboratoire) à celles d'autres types de population qui sont soit plus directement concernés au quotidien par le phénomène étudié (des managers pour étudier la performance de différents mécanismes incitatifs, par exemple) soit appartenant à des catégories socio-professionnelles plus variées en termes de revenu, d'occupation ou de statut marital (voir Fréchette [2009] pour une revue de littérature).

Le dernier avantage majeur des méthodes expérimentales est la modestie de leur coût financier. Si la mise en place d'un laboratoire fait l'objet d'un coût fixe (infrastructure physique et logicielle, investissement en méthodes), le recours à l'expérimentation en laboratoire reste, dans le cadre des pratiques actuelles, une technique économe et rapide d'obtenir des données et des régularités empiriques. Ce faible coût est d'un intérêt particulier pour l'évaluation des politiques publiques puisqu'il permet de tester sur de petits échantillons des méthodes de mesure des préférences, des environnements de choix particuliers ou des hypothèses d'interactions sociales avant de lancer l'évaluation réelle à grande échelle. La limite intrinsèque de l'économie expérimentale tient en effet à la mesure dans laquelle elle est capable d'informer sur les comportements qui surviendraient à l'extérieur du laboratoire, dans les situations réelles que l'expérience s'efforce de répliquer.

\footnotetext{
${ }^{5}$ Pour mémoire, dans le jeu de l'ultimatum, deux joueurs interagissent pour décider de la division d'une somme. Le premier joueur propose une division de la somme et le second a le choix entre accepter ou rejeter cette offre. L'acceptation conduit au partage proposé par le premier joueur, le refus correspond au statu quo. L'équilibre parfait en sous jeu est une proposition de partage donnant la part la plus faible, mais non nulle, au second joueur, proposition acceptée par ce dernier. Les résultats expérimentaux montrent qu'en général, les propositions sont en fait de l'ordre de 20 à $50 \%$ de la somme. Ces résultats sont en général pris comme exemple de l'importance des préférences sociales des individus.
} 
1.3 Comportements expérimentaux et comportements réels: la validité externe des expériences

La capacité de contrôle offerte par les expériences a pour contrepartie directe de réduire les situations réelles à des environnements artificiels et décontextualisés qui conduisent à mettre en doute le parallélisme (Smith [1982]) entre les comportements observés dans le laboratoire et ceux qu'induirait le même environnement dans une situation réelle.

Cette question de la validité externe des résultats expérimentaux fait donc directement référence au lien entre l'observation fournie par la situation expérimentale, $F^{m}\left(x^{n}\right.$ $\left.U x^{\infty-n}\right)$ dans les termes des définitions développées précédemment, et les conséquences observées dans la réalité lorsque les mêmes déterminants sont à l'œuvre : $S^{m} \in S^{\infty}\left(x^{n} U X^{\infty-}\right.$ $\left.{ }^{n}\right)$. Cette définition met en évidence un premier élément important de la réflexion sur la validité externe des résultats expérimentaux. Comme indiqué plus haut, les expériences en laboratoire et la modélisation théorique opèrent toutes deux une réduction du réel destinée à isoler les mécanismes causaux d'intérêt. Par construction, et même par nature, la validité externe des expériences en laboratoire est donc en tout point identique à celle du modèle théorique qu'elle cherche à tester : la modélisation théorique, de même que les expériences en laboratoire, réduit les situations économiques d'intérêt à un faisceau étroit de causes et de conséquences afin de les rendre intelligibles; cette réduction ne peut que conduire à s'interroger sur la pertinence pratique du modèle théorique, sa capacité à prendre en compte les mécanismes à l'œuvre dans la situation d'intérêt. Lorsque les expériences visent à tester la théorie, la question de la validité externe s'adresse donc dans les mêmes termes à la compréhension du réel fournie par la théorie qu'aux observations issues de l'expérience.

S'agissant plus spécifiquement des expériences en laboratoire, les définitions décrites plus haut permettent de distinguer différents niveaux d'exigence en termes de validité externe. D'abord, une remise en cause de la validité externe proprement dite porte sur une éventuelle déconnexion entre l'observation issue du laboratoire d'une part, et d'autre part les conséquences induites par les mêmes inputs à l'extérieur du laboratoire. A ce premier niveau d'exigence, interroger la validité externe consiste donc à suspecter une distorsion de la relation causale d'intérêt en raison uniquement de l'artificialité de l'environnement de laboratoire. Une définition moins restrictive, et donc plus exigeante, consiste à se demander si les conséquences observées dans le laboratoire seraient également induites par des inputs similaires à ceux qui ont été choisis (par exemple, est ce que les comportements engendrés par une rémunération équivalente à une heure de travail 
payée au salaire minimum sont comparables, ou radicalement différents, de ceux qui seraient associés à des montants monétaires de l'ordre d'un salaire mensuel). A ce deuxième niveau d'exigence, la question de la validité externe est posée en termes d'inférence à un éventail plus large de situations des résultats obtenus dans le laboratoire : les comportements observés sont-ils informatifs de ceux qui seront adoptés dans des situations similaires par les agents économiques ? Cette définition est celle qui est la plus communément adoptée dans les discussions, et qui donne son sens à la terminologie : il s'agit de savoir si les relations causales identifiées dans le laboratoire restent valides à l'extérieur de l'environnement dans lequel elles ont été étudiées; si elles ont un degré minimum de généralité.

Poussée à son extrême, cette notion de validité externe peut aller jusqu'à être assimilée à la remise en cause de la robustesse des résultats expérimentaux. Cette question de la robustesse est évidemment commune à toutes les investigations empiriques, quelle qu'en soit la méthodologie. Elle consiste à identifier l'éventail des situations réelles auxquelles les résultats empiriques s'appliquent - en termes de population d'appartenance, de type de décision économique, etc. En ce sens, la question de la validité externe apparaît moins comme une limitation inhérente aux investigations empiriques en laboratoire, que comme une nécessité de dialogue entre la représentation de la réalité et l'environnement étudié dans le laboratoire, destiné à identifier le périmètre de concordance entre ces deux types de situations.

L'exploration de la validité externe des résultats expérimentaux se traduit ainsi par un processus incrémental, consistant à identifier un à un les éléments des situations réelles d'intérêt qui sont à la fois (i) négligés de l'expérience originelle ; et (ii) susceptibles d'affecter sensiblement les comportements. Sous l'impulsion notamment du recours croissant aux expériences de terrain ${ }^{6}$, cette direction de recherche s'est fortement intensifiée au tournant des années 2000, à travers la réalisation d'expériences (en laboratoire ou de terrain) qui répliquent des protocoles antérieurs dans des circonstances différentes. Leur objectif est d'évaluer la robustesse des régularités expérimentales les plus connues aux dimensions des expériences qui sont généralement considérées comme les plus limitatives sur leur degré de validité externe. Ainsi ont été étudiés tour à tour l'influence du biais de sélection dû à la population des participants aux expériences (dont l'immense majorité est généralement composée d'étudiants) ou de leur absence de familiarité avec la situation dans laquelle ils

\footnotetext{
${ }^{6}$ La discussion des aspects méthodologiques de ce type particulier d'expérience dépasse le champ du présent article. Harrison et List [2004], List [2006] ou encore Levitt et List [2009] proposent un survol de cette littérature.
} 
agissent (par opposition aux agents prenant les mêmes décisions dans les situations économiques correspondantes), du montant des sommes monétaires (souvent considérées comme trop modestes au regard des enjeux économiques de la situation réelle) ou encore de l'artificialité des tâches à valeur induite (qui permettent de contrôler parfaitement les paramètres de préférences sous-jacents aux décisions, mais au prix d'un environnement très abstrait). La recension exhaustive de l'ensemble de ces résultats constituerait (et mériterait) un article en soi. Mais trois leçons essentielles semblent se dégager des résultats accumulés au cours de la dernière décennie.

D'une part, les différentes dimensions citées ci-dessus (population de sujets, montants monétaires, etc.) ont pour principale conséquence d'affecter la dispersion des comportements, mais semblent avoir relativement peu d'influence à la fois sur les résultats qualitatifs, et surtout sur les comportements moyens ${ }^{7}$. Ensuite, la familiarité des participants avec la situation dans laquelle ils sont amenés à prendre une décision semble influencer les résultats uniquement à travers un processus d'apprentissage (plutôt qu'en raison de différences intrinsèques dans les déterminants du comportement entre les participants habituels des expériences et les personnes effectivement confrontées aux situations économiques étudiées). Enfin, les résultats expérimentaux qui mettent en évidence l'existence de préférences sociales (une préoccupation pour la situation des autres dans la prise de décision qui se démarque de l'hypothèse classique d'égoïsme pur des agents économiques), apparaissent très sensibles à la configuration de l'environnement dans lequel elles sont étudiées ${ }^{8}$.

\section{Un exemple d'évaluation : le cas des marchés d'appariement}

Dans cette section nous présentons un exemple d'évaluation de politiques publiques au travers de la question de l'appariement sur le marché du travail. Les marchés d'appariement constituent un exemple d'autant plus significatif de la reconnaissance de l'économie expérimentale comme outil d'évaluation des institutions du marché du travail qu'ils viennent de faire l'objet du prix Nobel d'économie, attribué conjointement en 2012 à

\footnotetext{
${ }^{7}$ Falk et Fehr [2003] proposent un survol de quelques exemples de travaux qui aboutissent à cette conclusion.

${ }^{8}$ Hoffman et al. [1994] ; Cherry et al. [2002] ; Bardsley [2008] comptent parmi les résultats les plus marquants à cet égard; voir notamment Levitt et List [2007] pour une revue de littérature (à charge) et Cooper et Kagel [2013] pour un survol historique.
} 
Lloyd Shapley, pour ses contributions théoriques, et à Alvin Roth, pour ses travaux empiriques.

\subsection{La question des marchés d'appariement}

La littérature sur les marchés d'appariement s'intéresse aux processus de recherche de partenaires lorsqu'il y a deux ensembles d'agents de chaque coté du marché et que chaque agent ne peut être apparié qu'avec un partenaire de l'autre coté du marché. Les exemples de telles situations concernent l'admission aux Universités américaines ou le mariage. Ces exemples sont ceux étudiés dans l'article fondateur de Gale et Shapley [1962] consacré aux processus d'appariement sur de tels marchés. Le marché du travail est un cadre naturel d'étude pour les marchés d'appariement (Shapley et Shubik [1971] ; Kelso et Crawford [1982]), en particulier pour l'affectation des médecins en fin d'études aux hôpitaux. La question initiale de cette littérature est de savoir si le mécanisme d'allocation entre agents génère des appariements qui sont stables. Un appariement entre un travailleur et un employeur est stable s'il n'existe aucune paire possible travailleur/employeur non appariée alors que chacun des partenaires préfèrerait entrer dans cet appariement. Au contraire, un appariement est instable si l'un des deux partenaires (ou les deux) préfère(nt) un autre appariement réalisable à son (leur) appariement actuel.

Une caractéristique économique importante des marchés d'appariement est leur tendance à être trop étroits et à souffrir d'externalités de congestion ${ }^{9}$ (Roth [2008]). En pratique, un nombre important de marchés d'appariement se caractérise par des dynamiques de transaction très particulières en réponse aux phénomènes de congestion. Pour bénéficier du marché le plus large possible, chacun a intérêt à choisir le plus tôt possible pour garder ses chances de trouver le bon partenaire. Les transactions ont alors tendance à se faire très à l'avance par rapport au moment où la paire sera effectivement productive. Roth et Xing [1994] ont montré que c'était le cas, par exemple, pour le recrutement des greffiers des juges fédéraux américains, des médecins britanniques ou encore des diplômés des meilleures universités japonaises. A ces exemples s'ajoutent ceux des avocats (Avery et al. [2001]) et des gastroentérologues aux Etats-Unis (Niederle et al. [2006]). Cette situation dite de «détricotage» (unraveling) est l'échec de marché majeur observé sur les marchés d'appariement dans la réalité. Le détricotage est susceptible

\footnotetext{
${ }^{9}$ Par exemple, lorsque qu'un employeur intensifie sa recherche d'employé, il réduit, toutes choses égales par ailleurs, les chances de ses homologues de s'apparier. De même l'entrée d'un employeur sur un marché d'appariement crée de la congestion et réduit les chances d'appariement de ses homologues.
} 
d'engendrer de nombreux coûts: perte de flexibilité de planification du fait du préengagement, qualité incertaine du partenaire par exemple. A ce titre, ce phénomène constitue un réel enjeu de politique publique. Dans une perspective d'évaluation de politiques publiques, la question se pose donc de savoir si le choix d'un mécanisme d'allocation, autre que le laissez-faire, permet d'éviter ce phénomène, tout en assurant des appariements stables. L'article de Kagel et Roth [2000] montre en quoi le recours à des méthodes expérimentales permet d'apporter des éléments de réponse à cette question. L'intérêt de la méthodologie expérimentale est de permettre d'étudier l'impact de différents types de mécanismes d'allocation, d'algorithmes d'affectation, en contrôlant parfaitement le reste de l'environnement de choix. Les observations expérimentales sont ainsi une démonstration de robustesse des observations effectuées avec des méthodes alternatives.

\subsection{L'environnement dans Kagel et Roth [2000]}

Habituellement, trois éléments essentiels constituent une expérience, l'environnement, les institutions et le cadrage. Le premier élément, l'environnement de choix dans lequel les participants se situent, est l'objet de cette sous-section. Comme dans l'analyse économique traditionnelle, l'environnement regroupe les préférences, la technologie de production, les dotations initiales. Le contrôle dont dispose l'expérimentateur pour agir sur cet environnement repose sur des incitations monétaires, qui permettent d'induire différents choix de partenaires et de spécifier différentes technologies.

Dans l'expérience de Kagel et Roth [2000], les participants sont regroupés par groupes de 12 personnes : la moitié jouant le rôle de firmes, l'autre moitié jouant le rôle de travailleurs. Au sein de chaque groupe de 6 personnes, 3 seront associées à des niveaux de productivité faible et 3 seront associées à des productivités élevées. L'assignation dans chaque groupe et sous-groupe est aléatoire. Les incitations dépendent de l'appariement réalisé par chacun: l'appariement avec un partenaire de haute productivité génère un paiement trois fois plus important que l'appariement avec un partenaire de basse productivité. Ces éléments sont communs à tous et de connaissance commune. Ces incitations montrent que chaque firme (resp. travailleur), quelle qu'elle soit, préfère un appariement avec un travailleur (resp. firme) de haute productivité plutôt qu'un appariement avec un travailleur (resp. firme) de basse productivité. De plus, chaque appariement possible génère pour chaque participant un paiement spécifique. Ces incitations spécifiques permettent d'obtenir une variabilité des préférences pour les partenaires au sein des sousgroupes de haute et basse productivités. La structure des incitations permet ainsi de 
contrôler les classements de partenaires dans l'expérience: les paiements communs assurent la dominance du classement par productivité (haute ou élevée), les paiements spécifiques assurent des classements préférés différents à l'intérieur de chaque sousgroupe.

\subsection{Les institutions dans Kagel et Roth [2000]}

Dans cette section, nous décrivons le deuxième élément essentiel d'une expérience : les institutions. Celles-ci regroupent l'ensemble des actions possibles pour chaque participant, la séquence possible de ses actions, ses interactions avec d'autres participants, les conditions informationnelles dont il dispose. L'expérimentateur contrôle ces institutions en spécifiant les règles du jeu.

Dans l'expérience de Kagel et Roth [2000], chaque marché d'appariement est constitué de trois périodes. Les appariements sont possibles au sein de chaque période, mais tout appariement réalisé avant la dernière période donne lieu à un coût fixe. Les actions possibles sont les suivantes : pour qu'un appariement soit réalisé, une firme doit faire une offre à un travailleur et cette dernière doit être acceptée par le travailleur. La séquence d'action est caractérisée par la possibilité de faire (resp. d'accepter) une offre dans les trois périodes. Tout appariement conclu est réputé stable et sort du marché. L'interaction entre les participants au marché est limitée à ce processus d'offre. Cette offre est ainsi très simple et ne porte que sur la volonté de s'apparier : les paiements reçus par chacun ne font l'objet ni d'affichage public (de «postage»), ni de négociation. En termes de conditions informationnelles, les différences de paiement haute/basse productivité sont de connaissance commune, les paiements spécifiques restent d'information privée dans un marché donné. De même les offres et les rejets restent d'information privée. Le choix des institutions permet ainsi de contrôler les conditions dans lesquelles les comportements sont observés. En raison du coût fixe, les paiements font en sorte que chacun (firme ou travailleur) a intérêt à s'apparier de manière stable à l'avance avec un partenaire de haute productivité, mais qu'à l'intérieur de chaque classe (de haute ou basse productivité) il n'est pas profitable de rechercher un partenaire à l'avance. Les institutions génèrent ainsi un échec de marché, car aucun participant n'a intérêt à une coordination en dernière période. Afin de comparer cette solution décentralisée inefficiente et deux mécanismes d'allocation, les sujets participent à 10 marchés décentralisés suivis de 15 marchés avec un mécanisme 
d'allocation particulier (algorithme de Gale-Shapley ou algorithme de Newcastle ${ }^{10}$ ), et ce, pour cinq structures de paiement différentes. Les sujets sont alloués au hasard au groupe confronté à chaque type de mécanisme d'allocation centralisé.

\subsection{Principaux résultats}

Les résultats montrent que si les entreprises se présentent trop tôt sur un marché décentralisé pour capter les travailleurs de haute productivité, les deux mécanismes d'allocation envisagés pour réduire l'inefficience sont loin d'être équivalents. L'introduction de l'algorithme de priorité de Newcastle ne change pas cette situation, alors que l'algorithme de Gale-Shapley réduit drastiquement le phénomène de détricotage. La séparation contrôlée entre travailleurs de haute productivité et de basse productivité montre que l'inefficience est principalement due à la volonté des firmes de capter ces compétences. Si l'algorithme de Gale-Shapley permet à ces travailleurs d'accéder aux emplois désirés dans des conditions socialement efficaces, seul le recours à des stratégies d'avancement d'embauche permet de réaliser les appariements individuellement souhaités dans l'algorithme de Newcastle, pour échapper aux effets de congestion. Hormis la démonstration de la robustesse de ce résultat observé dans les appariements entre médecins et hôpitaux, l'expérience de Kagel et Roth [2000] permet d'observer de manière fine et contrôlée les phénomènes de transition dans les comportements observés de chacun des types de participants. A cet égard, les méthodes expérimentales apportent un éclairage complémentaire précieux dans la démarche d'évaluation des politiques publiques, en complétant les observations ou estimations réalisées ex post par des observations sur des dynamiques inobservables par ailleurs, et en évitant de mettre en œuvre des évaluations par affectation aléatoire pour étudier des interactions basiques entre les comportements et les institutions.

\section{L'approche expérimentale de la prospection d'emploi}

Les modèles de prospection sont à la base des modèles canoniques de représentation du marché du travail que sont le modèle de Mortensen et Pissarides [1999] et le modèle de Burdett et Mortensen [1998]. Ces modèles, du fait de leur aspect dynamique intégrant les processus de création et de destruction d'emploi ainsi que la notion de

\footnotetext{
${ }^{10}$ L'algorithme de Gale-Shapley est un algorithme de rejet/acceptation tandis que l'algorithme de Newcastle est un algorithme dit de priorité. Ces algorithmes sont présentés en Annexe.
} 
distribution de salaires, ont été utilisés comme un outil de tout premier plan pour l'analyse d'impact des politiques de l'emploi, notamment en ce qui concerne les allocations chômage (Cahuc et Zylberberg [2004] ; Daly et al. [2012] ; Fontaine et Malherbet [2013]). Dans cette partie, nous présentons tout d'abord les protocoles expérimentaux avant de nous tourner vers les principaux résultats de la littérature et le lien avec les politiques du marché du travail.

\subsection{Modèles de prospection et protocoles expérimentaux}

Les modèles de prospection fournissent de nombreuses prédictions quant aux comportements de recherche des demandeurs d'emploi. Cependant, pour constituer une représentation pertinente des comportements, ces modèles doivent être fondés empiriquement. En particulier, dans ces modèles, le comportement optimal est caractérisé par une règle d'arrêt, également nommée «propriété de salaire de réservation » dans la littérature expérimentale. Le comportement d'un individu en recherche d'emploi consiste à continuer à chercher un emploi jusqu'à ce qu'il reçoive une offre de salaire supérieure ou égale à son salaire de réservation ${ }^{11}$. En termes comportementaux, une première question émerge alors : les demandeurs d'emploi se comportent-ils conformément à cette règle d'arrêt optimale ? Les études empiriques sur données réelles, qui représentent l'outil dominant dans l'analyse des comportements sur le marché du travail, peuvent difficilement répondre à cette question dans la mesure où les salaires de réservation des demandeurs d'emploi ne sont pas observés. De même, l'information sur la distribution des offres, l'horizon de recherche et la possibilité d'accéder à des offres passées, qui sont des déterminants essentiels des comportements optimaux de recherche, ne sont pas aisément identifiables dans les données d'enquête ou les données administratives. A cet égard, la méthode expérimentale apparaît comme un outil complémentaire intéressant pour tester les modèles de prospection puisqu'elle n'est pas soumise aux précédentes limites.

\footnotetext{
${ }^{11}$ Le salaire de réservation est défini tel que le gain marginal espéré d'une activité de recherche supplémentaire (par exemple une période supplémentaire de recherche) soit égal au coût marginal de cette recherche.
} 


\section{Encadré 1}

Un exemple d'expérience de laboratoire : l'expérience de Cox and Oaxaca [1989]

Les participants à l'expérience de Cox et Oaxaca [1989] sont des étudiants de l'université d'Arizona. A son arrivée au laboratoire, chaque participant reçoit un document décrivant les règles de l'expérience. Ces instructions sont lues par l'expérimentateur, qui répond également aux éventuelles questions du sujet. Le participant est informé que l'expérience comprend plusieurs séquences de recherche, que les règles qu'il vient de recevoir correspondent à la première séquence et qu'il recevra ultérieurement les instructions relatives aux autres séquences.

La première séquence comprend 20 périodes, qui se déroulent toutes de manière identique. Au début de chaque période, le sujet participe à un premier tirage au sort. Ce tirage est réalisé dans une urne contenant 2 boules blanches et 2 boules noires. Si le sujet tire une boule noire il ne reçoit pas d'offre de travail à cette période et il commence immédiatement la période suivante qui se déroule de la même manière. Inversement, si le sujet tire une boule blanche il reçoit une offre de travail et il participe à un second tirage au sort. Ce second tirage fixe la valeur du salaire offert au sujet. II est réalisé dans une urne contenant 10 boules numérotées de 1 à 10 . A la suite de ce tirage, le sujet doit choisir entre accepter ou refuser l'offre de salaire qu'il vient de tirer. S'il refuse l'offre, il continue sa recherche en passant à la période suivante qui se déroule de manière identique. En revanche, si le participant accepte l'offre il reçoit le montant indiqué sur la boule tirée au sort pour toutes les périodes restantes dans la séquence de recherche. Son gain pour cette séquence de recherche s'élève donc au chiffre indiqué sur la boule tirée au sort lors du second tirage multiplié par le nombre de périodes restantes dans la séquence. Dans ce cas, le participant a terminé cette séquence de recherche et il passe à la suivante.

Les différentes séquences correspondent à autant de traitements qui permettent de faire varier les paramètres de l'expérience, comme par exemple le nombre de boules dans chaque urne ou le nombre de périodes de jeu. Au début de chaque séquence, le participant est informé des changements opérés. Dans l'expérience de Cox et Oaxaca [1989], les tirages sont réels, et non informatisés, et ils sont réalisés par le sujet lui-même. Avant chaque tirage, le participant peut contrôler le contenu de l'urne. Pour s'assurer que le participant est bien compris les règles de l'expérience et pour lui permettre de se familiariser avec le jeu, il participe tout d'abord à des séquences d'essai qui ne sont pas prises en compte dans le calcul de son gain. A la fin de l'expérience, le participant reçoit sa rémunération calculée à partir de la somme des gains qu'il a accumulé au cours de toutes

Les premières expériences sur les comportements de recherche d'emploi ont été réalisées par Braunstein et Schotter [1981] ; [1982] auprès d'étudiants. Leur protocole expérimental reproduit le modèle de base à horizon infini (Lippman et McCall [1976a] ; [1976b]). A chaque période, le sujet reçoit une offre tirée d'une distribution d'offres de salaires connue. Chaque offre est coûteuse et le nombre d'offres que le participant peut recevoir est illimité. Pour chaque offre, la décision du sujet consiste soit à accepter l'offre proposée et recevoir le montant actualisé correspondant soit à refuser l'offre et à poursuivre 
sa recherche ${ }^{12}$. II est important de noter que ce protocole expérimental, comme l'ensemble des travaux expérimentaux sur les comportements de recherche, fournit une représentation simplifiée du processus de recherche d'emploi. En particulier, il n'intègre pas les coûts liés à l'offre de travail, comme la perte de loisir ou la pénibilité du travail. En effet, les participants sont contraints de chercher et n'arbitrent donc pas entre loisir et travail. De plus, l'expérience se limite à la période de recherche et prend fin dès que le participant accepte une offre sans tenir compte des coûts futurs liés à l'effort au travail ${ }^{13}$.

A la suite de ces premières investigations, Cox and Oaxaca [1989] ; [1992] ; [1996] ; [2000] ont proposé une série d'expériences dont la principale différence avec les travaux de Braunstein et Schotter [1981] ; [1982] est l'introduction d'un horizon fini de recherche. Cox et Oaxaca [1989] argumentent qu'un horizon de recherche infini n'est pas crédible dans une expérience en laboratoire et que les comportements observés doivent, par conséquent, être analysés à partir d'un modèle de prospection à horizon fini. Cette différence n'est pas négligeable car elle altère profondément les prédictions issues du modèle de prospection. Alors que le salaire de réservation optimal est constant dans un environnement stationnaire avec horizon de recherche infini, et ce quel que soit le degré d'aversion au risque des agents ${ }^{14}$, cette propriété n'est plus vérifiée lorsque l'on introduit un horizon fini.

Du fait de sa plus grande cohérence avec les méthodes habituelles de l'économie expérimentale, le protocole de Cox et Oaxaca [1989] est devenu une référence incontournable pour l'évaluation des modèles de prospection d'emploi. Ce dernier, qui est décrit en détail dans l'encadré 1, a fait l'objet de nombreuses variantes afin de déterminer si la réaction des individus aux changements des institutions est conforme à la théorie. A cet égard, l'expérience de Cox et Oaxaca [1989] est composée de plusieurs traitements permettant de faire varier successivement le taux d'intérêt, les coûts et les subventions à la recherche, la distribution des offres de salaire, le taux d'arrivée des offres et la fin de l'horizon de recherche. La possibilité d'avoir accès aux offres passées a également été

\footnotetext{
12 D'autres expériences ont également été réalisées pour étudier les stratégies de recherche (Hey [1981] ; [1982] ; [1987] et Sonnemans [1998]) et l'intensité de la recherche (Harrison et Morgan [1990]) mais ces dernières ne sont pas présentées dans cet article.

${ }^{13}$ Des travaux expérimentaux s'intéressent à l'arbitrage entre loisir et travail en permettant notamment aux sujets de lire des magazines ou d'aller sur Internet pendant l'expérience (Eriksson et al. [2009] ; Corgnet, et al. [2013]). Mais ces travaux portent principalement sur des questions d'organisation du travail et de schéma de rémunération et non sur les comportements de recherche d'emploi.

${ }^{14}$ Dans un environnement stationnaire avec horizon de recherche infini, le salaire de réservation reste constant même en relâchant l'hypothèse de neutralité vis-à-vis du risque. La prise en compte de l'aversion pour le risque diminue la valeur du salaire de réservation optimal mais n'implique pas une décroissance de ce dernier au cours des périodes.
} 
analysée (Cox et Oaxaca [1996]) ${ }^{15}$. Les résultats de ces expériences sont conformes aux principales prédictions des modèles de prospection.

Cox et Oaxaca [2000] s'intéressent également aux effets de l'incertitude quant à la distribution des offres. Dans les traitements avec incertitude, les offres de salaire sont tirées aléatoirement de deux distributions d'offre de salaire, l'une étant plus «favorable " que l'autre. Ces deux distributions sont de connaissance commune mais les sujets ne savent pas de quelle distribution les offres de salaire sont effectivement tirées. La seule information dont ils disposent est la probabilité que chaque distribution soit retenue pour effectuer les tirages aléatoires des offres. Cette modification expérimentale est également capitale puisqu'elle conduit à des situations où la propriété de salaire de réservation n'est plus vérifiée : dans une même période un agent qui se comporte de manière optimale devrait accepter certaines offres alors qu'elles sont plus faibles que certaines offres qu'il devrait rejeter. Ce résultat s'explique par le changement d'environnement lié à l'introduction de l'incertitude : chaque offre faite contient ici une information sur la distribution des offres et les individus intègrent cette information en révisant leurs croyances. Ainsi, une offre de salaire élevée tend à indiquer que les offres proviennent de la distribution la plus favorable alors qu'une offre de salaire faible semble signaler qu'au contraire, les offres proviennent de la distribution moins favorable.

Plus récemment, Brown et al. [2011] proposent une expérience originale. Comme Braunstein et Schotter [1981] ; [1982] ils considèrent un environnement stationnaire avec horizon de recherche infini, mais ils proposent un jeu expérimental de prospection en temps réel et non plus sur un nombre fixe de périodes. Le temps est mesuré en secondes et les offres arrivent à un taux déterminé par une distribution de Poisson. Brown et al. [2011] s'intéressent aux effets du temps écoulé et des coûts accumulés au cours du processus de recherche. Ces deux éléments étant colinéaires dans leur expérience, ils conduisent deux traitements pour isoler leur impact respectif. Le premier traitement élimine le temps d'attente entre les offres mais maintient les coûts de recherche. Le second traitement conserve un taux d'arrivée des offres aléatoire mais fixe des coûts de recherche nuls.

\footnotetext{
${ }^{15}$ Selon les traitements expérimentaux, les sujets ont accès seulement à l'offre présente ou à l'ensemble des offres passées avec certitude ou aux offres passées avec une probabilité qui dépend négativement du nombre de périodes écoulées entre l'offre passée et la période actuelle.
} 


\subsection{Principaux résultats expérimentaux}

Alors que les jeux expérimentaux de prospection impliquent des décisions individuelles beaucoup plus complexes que la plupart des expériences de choix individuels, les résultats sont étonnamment favorables à la validité des prédictions théoriques des modèles de recherche d'emploi.

Les résultats des expériences à horizon infini (Braunstein et Schotter [1981] et Brown et al. [2011]) révèlent que les comportements de recherche sont globalement cohérents avec la théorie. D'une part, les salaires de réservation déclarés par les participants au début du processus de recherche ne sont pas différents de la valeur optimale prédite par le modèle (Brown et al. [2011]). D'autre part, les différentes variations de l'environnement de choix modifiant par exemple la distribution des offres ou les coûts de recherche, produisent les effets attendus sur les décisions de prospection (Braunstein et Schotter [1981]) ${ }^{16}$. Cependant, un résultat majeur du modèle de recherche en horizon infini est réfuté : le salaire de réservation n'est pas constant, il diminue au cours du processus de recherche. Cox et Oaxaca [1989] attribuent ce résultat au fait que l'horizon de recherche infini n'est pas crédible dans une expérience en laboratoire. Les participants conscients de cette contrainte se seraient alors comportés comme dans un jeu à horizon fini, expliquant ainsi la décroissance du salaire de réservation.

Les résultats des expériences à horizon fini (Cox et Oaxaca [1989] ; [2000]) montrent que les durées de recherche observées sont également conformes à la théorie quelles que soient les institutions mises en place. Néanmoins, parmi les décisions qui diffèrent de la stratégie optimale (23\%), les durées de recherche observées tendent à être plus courtes que celles prédites par la théorie (Cox et Oaxaca [1989]) $)^{17}$. II y a donc une légère tendance à arrêter la prospection trop rapidement. Ces résultats sont robustes même en présence d'incertitude quant à la distribution des offres de salaire.

L'observation des durées de recherche ne permet cependant pas de tester avec précision l'existence d'un salaire de réservation sur lequel se fonde la règle d'arrêt de la recherche puisque celui-ci n'est pas observé directement. En effet, différents salaires de réservation peuvent conduire à la même durée de recherche, cette dernière étant fortement conditionnée par les tirages aléatoires des offres de salaire. En utilisant une procédure de

\footnotetext{
${ }^{16}$ Les effets des institutions sont développés dans l'ouvrage de Cahuc et Zylberberg [2004].

${ }^{17}$ Parmi les participants qui n'arrêtent pas la recherche de manière optimale, les 3/4 arrêtent trop tôt alors que seulement $1 / 4$ arrête trop tard.
} 
révélation ${ }^{18}$, Cox and Oaxaca [1992] montrent que les individus fixent en moyenne des salaires de réservation inférieurs à la valeur optimale. Ce constat est concordant avec les précédents résultats expérimentaux sur la tendance à arrêter prématurément la recherche. Cox and Oaxaca [1992] attribuent ce résultat à l'aversion au risque et concluent qu'un modèle avec aversion au risque explique mieux les comportements observés qu'un modèle avec neutralité vis-à-vis du risque. Pour mémoire, dans un modèle standard de prospection d'emploi, le salaire de réservation d'un agent averse au risque est inférieur à celui d'un agent neutre au risque. Ce résultat s'applique au modèle à horizon fini où le salaire de réservation décline au cours des périodes, mais également au modèle à horizon infini où le salaire de réservation reste constant. En d'autres termes, dans un modèle à horizon infini, l'aversion pour le risque affecte le niveau du salaire de réservation de l'agent mais non sa dynamique au cours du processus de recherche.

Une précision doit être apportée quant à l'introduction d'un horizon de recherche fini. La présence d'un horizon fini relâche I'hypothèse de stationnarité du modèle de prospection mais n'explique pas pour autant la décroissance du salaire de réservation observée par Braunstein et Schotter [1981] dans un environnement stationnaire. Brown et al. [2011] tentent d'expliquer ce résultat en éliminant, à travers leur protocole expérimental, les sources de non stationnarité ${ }^{19}$. Dans ce cadre, deux explications comportementales peuvent justifier la baisse du salaire de réservation. La première est que les individus font une erreur de raisonnement en ne considérant pas les coûts accumulés de la recherche comme des coûts irrécupérables. Les individus cherchent donc à récupérer le plus possible ces coûts par leurs bénéfices futurs («sunk cost fallacy », Arkes et Blumer [1985]). La seconde est que les individus répondent au temps de recherche qui s'écoule car ils supportent un coût d'attente subjectif qui n'est pas pris en compte de manière explicite dans les modèles de prospection. Leurs résultats confirment la baisse du salaire de réservation dans un environnement stationnaire et montrent que seul le temps d'attente entre les offres reçues est capable d'expliquer la baisse de salaire observée. En généralisant, le temps d'attente serait alors la principale caractéristique du processus dynamique de recherche qui explique la non stationnarité des décisions des demandeurs d'emploi.

\footnotetext{
${ }^{18}$ La décision des sujets ne consiste plus à choisir entre accepter ou refuser les offres qui leur sont proposées mais ils doivent à chaque période indiquer leur salaire de réservation.

${ }^{19}$ Plusieurs explications théoriques ont été proposées pour expliquer le déclin du salaire de réservation mais elles relâchent toutes l'hypothèse de stationnarité. Par exemple Gronau [1971] considère un horizon fini de recherche ; Van den Berg [1990], Albrecht et Vroman [2005] introduisent des allocations chômage de court terme ; Danforth [1979] et Rendon [2006] considèrent des contraintes de liquidité ; Burdett et Vishwanath [1988] modélisent l'apprentissage quant à la distribution des offres.
} 


\subsection{Comportements et politiques du marché du travail}

Les expériences visant à tester les modèles de prospection d'emploi montrent que la grande majorité des comportements observés sont conformes aux prédictions théoriques, reposant pourtant sur des modèles où les hypothèses comportementales sont relativement fortes en termes de rationalité et de capacité de calcul des agents. En effet, le modèle de prospection d'emploi repose sur des hypothèses comportementales fortes: révision bayésienne des croyances, absence de myopie, traitement linéaire des probabilités, hypothèses largement remises en question par les études expérimentales précisément consacrées au sujet (Denant-Boëmont et l'Haridon [2013]). II est dès lors assez remarquable que les individus se comportent dans des processus de recherche suivant des règles très proches de celles prédites par le modèle. Ce résultat confirme et renforce la pertinence de l'utilisation de ces modèles pour comprendre mais également prédire les comportements de prospection des demandeurs d'emploi. De ce fait, ces modèles représentent un outil primordial pour l'analyse ex ante des politiques publiques sur le marché du travail. Mais la méthode expérimentale peut également être appliquée pour tester directement l'impact de telles politiques tout en gardant à l'esprit les réserves habituelles quant à l'interprétation des résultats expérimentaux.

Braunstein et Schotter [1982] testent les effets de l'introduction d'une offre minimum, sous la forme d'une borne inférieure dans la distribution des salaires. Pour ce faire, ils utilisent une distribution d'offre de salaire tronquée à gauche. Leurs résultats révèlent que la présence d'un salaire palier en dessous duquel il est impossible de descendre accroît significativement les salaires de réservation déclarés par les participants. Suite à l'introduction d'un palier d'un montant équivalant à $40 \%$ du salaire de réservation optimal, les participants augmentent leur salaire de réservation d'environ 10\%. Leur méthode pour simuler l'introduction de cet analogue de salaire minimum présente cependant deux inconvénients majeurs. D'une part, tronquer la distribution des offres altère la moyenne et la variance de la distribution ce qui rend difficile l'interprétation des résultats. D'autre part, leur protocole expérimental ne prend pas en compte le fait que sur le marché du travail tous les demandeurs d'emploi ne tirent pas leurs offres de la même distribution de salaires et donc ne sont pas affectés de la même manière par l'introduction d'un salaire minimum.

L'impact des allocations chômage a également fait l'objet d'études expérimentales (Braunstein et Schotter [1982] ; Cox et Oaxaca [1989]). Elles sont représentées par une subvention à la recherche versée à chaque période. A cet égard, l'expérience de Braunstein et Schotter [1982] semble plus adaptée que celle de Cox et Oaxaca [1989] car ils 
considèrent une subvention temporaire limitée à cinq périodes. Les résultats de ces deux expériences révèlent que l'introduction d'allocations chômage conduit à un accroissement des salaires de réservation et donc des durées de recherche. Ils montrent également que lorsque les sujets ont la possibilité d'accéder aux offres passées ils attendent jusqu'à la dernière période subventionnée avant d'accepter une offre. Les sujets consomment donc l'intégralité de leurs allocations avant d'intégrer le marché du travail.

Dans un article récent, Boone et al. [2009] s'intéressent aux allocations chômage couplées à d'éventuelles sanctions en cas de refus d'une offre. Dans leur protocole, un sujet qui accepte une offre est employé pour une période donnée puis il retourne ensuite au processus de recherche. II perçoit alors l'intégralité de l'allocation chômage pour la période qui suit la phase d'emploi. En revanche, dès qu'il refuse une offre de salaire il prend le risque d'être sanctionné à la période suivante et de voir ainsi le montant de son allocation diminuer. La sanction arrive avec une probabilité donnée qui est de connaissance commune. Leurs résultats montrent que les sanctions accélèrent le retour à l'emploi. Suite à l'introduction de sanctions allant de $25 \%$ à $75 \%$ du montant des allocations chômage, le taux de retour à l'emploi augmente en moyenne de $28 \%$. De plus, l'introduction de sanctions produit deux effets distincts. D'une part, les sujets acceptent plus rapidement une offre après avoir reçu une sanction (effet ex post). D'autre part, l'introduction d'une éventuelle sanction agit comme une menace et conduit les sujets à réduire leur durée de recherche avant même d'être sanctionnés (effet ex ante). L'ampleur de l'effet ex ante est supérieure à celle de l'effet ex post.

Les études expérimentales visant à analyser les effets des politiques de l'emploi sont encore peu nombreuses. Ces premiers résultats ouvrent la voie à de nombreuses directions de recherche destinées à en affiner la portée, par exemple en étudiant différents schémas de dégressivité des allocations chômage ou encore en liant le montant des allocations au salaire antérieur. De nombreuses politiques de l'emploi peuvent être répliquées et évaluées en laboratoire. II est, par exemple, possible de concevoir un protocole expérimental avec un marché du travail composé de plusieurs secteurs et d'étudier ainsi comment l'information disponible sur les caractéristiques des différents secteurs affecte les comportements de recherche. 


\section{Conclusion}

Dans cet article, nous avons présenté, au travers d'un cadre général d'analyse et d'exemples d'applications, l'intérêt de l'économie expérimentale pour l'évaluation des politiques publiques sur le marché du travail. Cet intérêt tient à la position particulière de l'économie expérimentale, qui se situe à l'intersection entre les réalités économiques, la modélisation théorique et l'observation empirique. L'apport et les limites de l'économie expérimentale reposent sur la tension permanente entre sa capacité de contrôle très forte sur l'environnement des décisions et la perte de réalisme associée à l'expérimentation.

L'exemple des marchés d'appariement illustre comment la méthode expérimentale permet non seulement de confirmer la robustesse des observations empiriques traditionnelles, mais également d'observer de manière fine et contrôlée les phénomènes de transition habituellement inobservables. L'exemple des marchés de prospection montre que la méthode expérimentale permet de tester la robustesse d'un modèle théorique mais également d'observer des variables inobservables, ou difficilement observables, dans les réalités économiques. A ce titre, l'économie expérimentale apporte deux éléments importants pour l'évaluation des politiques publiques du marché du travail. En premier lieu, et contrairement à ce que l'on pouvait attendre étant donnés les nombreux biais de rationalité observés en laboratoire, les études expérimentales valident le modèle de prospection d'emploi, largement utilisé dans l'évaluation et la conception des politiques publiques comme représentation des comportements individuels. En second lieu, la méthode expérimentale fournit de précieuses informations sur la forme des distributions des variables inobservables telles que les salaires de réservation et les intensités de recherche. 


\section{Annexe \\ Les algorithmes d'allocation centralisée utilisés par Kagel et Roth [2000]}

L'algorithme de Newcastle est un algorithme dit de priorité. Dans cet algorithme, chaque partenaire range les alternatives. Une priorité est alors définie pour chaque paire firme/travailleur en fonction de leurs rangs mutuels. Par exemple, une paire pour laquelle une firme est classée 1 par le travailleur et le travailleur classé 1 par la firme, sera de rang 1 , si la firme est classée 2 par le travailleur, la paire sera de rang 2. Une paire composée d'une firme classée 4 par le travailleur et d'un travailleur classé 4 par la firme, sera de rang 16 . L'algorithme apparie ainsi tous les couples de priorité 1 , puis les couples de priorité 2 , et ainsi de suite.

A titre d'illustration, supposons six firmes F1, F2, F3, F4, F5 et F6 à apparier avec six travailleurs T1, T2, T3, T4, T5 et T6. Chaque participant a classé les partenaires potentiels par ordre de préférence. Le tableau 1 présente ces classements. Dans chaque case, on trouve un couple de valeur: la première valeur indique le rang donné par la firme à ce travailleur, la seconde valeur indique le rang donné par le travailleur à cette firme. Par exemple, la première case, indique que la firme $\mathrm{F} 1$ classe en quatrième position le travailleur $\mathrm{T} 1$ et que le travailleur $\mathrm{T} 1$ classe la firme $\mathrm{F} 1$ en première position.

\begin{tabular}{|l|l|l|l|l|l|l|}
\hline & T1 & T2 & T3 & T4 & T5 & T6 \\
\hline F1 & $(1,1)$ & $(2,2)$ & $(3,3)$ & $(4,4)$ & $(1,5)$ & $(3,6)$ \\
\hline F2 & $(2,1)$ & $(1,3)$ & $(4,2)$ & $(3,4)$ & $(2,5)$ & $(2,6)$ \\
\hline F3 & $(3,3)$ & $(3,4)$ & $(2,1)$ & $(1,2)$ & $(4,5)$ & $(4,6)$ \\
\hline F4 & $(4,3)$ & $(4,4)$ & $(1,2)$ & $(2,1)$ & $(5,5)$ & $(5,6)$ \\
\hline F5 & $(5,1)$ & $(5,2)$ & $(5,4)$ & $(5,5)$ & $(3,3)$ & $(1,6)$ \\
\hline F6 & $(6,3)$ & $(6,1)$ & $(6,4)$ & $(6,5)$ & $(6,2)$ & $(6,6)$ \\
\hline
\end{tabular}

Tableau 1: un exemple de problème d'affectation de paires. Chaque paire possible firme/travailleur présente les classements respectifs sous la forme: (classement de la firme, classement du travailleur). 
L'algorithme de Newcastle classe les paires en calculant un score égal au produit des classements joints (c'est à dire au produit des deux nombres pour chaque parenthèse). Les rangs ainsi évalués sont donnés dans le tableau 2 :

\begin{tabular}{|l|l|l|l|l|l|l|}
\hline & T1 & T2 & T3 & T4 & T5 & T6 \\
\hline F1 & $\mathbf{1}$ & 4 & 9 & 16 & 5 & 18 \\
\hline F2 & 2 & $\mathbf{3}$ & 8 & 12 & 10 & 12 \\
\hline F3 & 9 & 12 & 2 & $\mathbf{2}$ & 20 & 24 \\
\hline F4 & 12 & 16 & $\mathbf{2}$ & 2 & 25 & 30 \\
\hline F5 & 5 & 10 & 20 & 25 & 9 & $\mathbf{6}$ \\
\hline F6 & 18 & 6 & 24 & 30 & $\mathbf{1 2}$ & 36 \\
\hline
\end{tabular}

Tableau 2 : les rangs dans l'algorithme de Newcastle.

L'affectation est alors réalisée dans l'ordre des rangs : la paire de rang 1 est la paire $(T 1, F 1)$, les paires de rang 2 sont $(\mathrm{T} 4, \mathrm{~F} 3)$ et $(\mathrm{T} 3, \mathrm{~F} 4)$ ou $(\mathrm{T} 3, \mathrm{~F} 3)$ et $(\mathrm{T} 4, \mathrm{~F} 4)$, la paire de rang 3 est la paire $(\mathrm{T} 2, \mathrm{~F} 2)$. Les deux dernières paires sont les paires de rang $6,(\mathrm{~T} 6, \mathrm{~F} 5)$ et la paire de rang 12, (T5,F6). Cet appariement n'est pas stable : le travailleur T5 préfèrerait être apparié à la firme $\mathrm{F} 5$, et réciproquement. II existe donc un appariement mutuellement avantageux entre le travailleur T5 et la firme F5 qui n'est pas exploité. Tous deux ont intérêt à s'apparier avant la centralisation pour éviter cette situation sous-optimale.

L'algorithme de Gale-Shapley est un algorithme d'acceptation/rejet. Dans cet algorithme, chaque travailleur classe les firmes correspondant à ses choix possibles. Tant qu'un travailleur n'est pas apparié, l'algorithme choisit une firme à laquelle le travailleur n'a pas été encore confronté. Si la firme est libre, alors l'appariement est réalisé. Si la firme est déjà appariée et qu'elle préfère le nouveau travailleur, alors elle est appariée avec lui. Sinon, la firme rejette le travailleur et une nouvelle firme sur la liste du travailleur entre à son tour dans le processus d'appariement. L'algorithme se poursuit jusqu'à que tous les travailleurs soient appariés.

Supposons dans l'exemple que les travailleurs T1, T2,T3,T4 et T6 soient provisoirement appariés aux firmes F1, F2, F3, F4 et F5. Nous supposons également que les travailleurs ont l'initiative des propositions. 
L'algorithme de Gale-Shapley pose qu'un travailleur libre, T5 va proposer à la firme F1 l'appariement. Celle-ci refuse car elle préfère son appariement actuel. Le travailleur propose alors à la firme F2, qui refuse également. Le travailleur se tourne vers la firme F5, son troisième choix, qui accepte et libère le travailleur T6. Le travailleur libre, désormais le travailleur T6, propose alors à la firme F5 qui refuse. Puis, dans l'ordre, aux firmes F2, F1, F4 et F4 qui toutes refusent. Reste, la firme F6, qui, actuellement non appariée, accepte.

Toutes les paires étant appariées, l'algorithme se termine. L'affectation est donc $(\mathrm{T} 1, \mathrm{~F} 1)$, (T2,F2), (T3,F3), (T4,F4), (T5,F5) et (T6,F6). Cet appariement est stable.

\section{Bibliographie}

J. Albrecht et S. Vroman [2005] : Equilibrium Search With Time-Varying Unemployment Benefits*, The Economic Journal 115, n505, pp.631-648.

J. Anderson, S. Burks, C. DeYoung, et A. Rustichini [2011] : Toward The Integration Of Personality Theory And Decision Theory In The Explanation Of Economic Behavior, Document de travail.

H.R. Arkes et C. Blumer [1985] : The Psychology Of Sunk Cost, Organizational behavior and human decision processes $35, n^{\circ} 1, p p .124-140$.

C. Avery, C. Jolls, R.A. Posner, et A.E. Roth [2001] : The Market For Federal Judicial Law Clerks, The University of Chicago Law Review pp.793-902.

O. Bandiera, I. Barankay, et I. Rasul [2010] : Social Incentives In The Workplace, The Review of Economic Studies 77, $n^{\circ} 2$, pp.417-458.

N. Bardsley [2008] : Dictator Game Giving: Altruism Or Artefact?, Experimental Economics 11, $\mathrm{n}^{\circ} 2$, pp.122-133.

G.J. Van den Berg [1990] : Nonstationarity In Job Search Theory, The review of economic studies $57, \mathrm{n}^{\circ} 2$, pp.255-277.

J. Boone, A. Sadrieh, et J.C. Van Ours [2009] : Experiments On Unemployment Benefit Sanctions And Job Search Behavior, European Economic Review 53, n8, pp.937-951.

Y.M. Braunstein et A. Schotter [1982] : Labor Market Search: An Experimental Study, Economic Inquiry 20, $\mathrm{n}^{\circ} 1, \mathrm{pp} .133-144$.

M. Brown, C.J. Flinn, et A. Schotter [2011] : Real-time Search In The Laboratory And The Market, The American Economic Review 101, n², pp.948-974.

K. Burdett et T. Vishwanath [1988] : Declining Reservation Wages And Learning, The Review of Economic Studies 55, n4, pp.655-665.

P. Cahuc et A. Zylberberg [2004] : Labor Economics. MIT Press.

C. Camerer [2003] : Behavioral game theory: experiments in strategic interaction. Princeton University Press.

G. Charness et P. Kuhn [2011] : Lab Labor: What Can Labor Economists Learn From The Lab?, Handbook of Labor Economics 4, pp.229-330. 
T.L. Cherry, P. Frykblom, et J.F. Shogren [2002] : Hardnose The Dictator, American Economic Review 92, n4, pp.1218-1221.

M. Coller et M.B. Williams [1999] : Eliciting Individual Discount Rates, Experimental Economics 2, n², pp.107-127.

D. Cooper et J. Kagel [2013] : Handbook Of Experimental Economics.

B. Corgnet, R. Hernean-Gonzalez et E. Schniter [2013], Why Real Leisure Really Matters: Incentive Effects on Real Effort in the Laboratory, Document de travail.

J.C. Cox et R.L. Oaxaca [2000] : Good News And Bad News: Search From Unknown Wage Offer Distributions, Experimental Economics 2, n³, pp.197-225.

J.C. Cox et R.L. Oaxaca [1996] : Testing Job Search Models: The Laboratory Approach, Research in labor economics 15, pp.171-207.

J.C. Cox et R.L. Oaxaca [1992] : Direct Tests Of The Reservation Wage Property, The Economic Journal 102, n415, pp.1423-1432.

J.C. Cox et R.L. Oaxaca [1989] : Laboratory Experiments With A Finite-horizon Job-search Model, Journal of Risk and Uncertainty 2, n³, pp.301-329.

R. Croson et U. Gneezy [2009] : Gender Differences In Preferences, Journal of Economic Literature pp.448-474.

M.C. Daly, B. Hobijn, A. Şahin, et R.G. Valletta [2012] : A Search And Matching Approach To Labor Markets: Did The Natural Rate Of Unemployment Rise?, The Journal of Economic Perspectives 26, n`3, pp.3-26.

J.P. Danforth [1979] : On The Role Of Consumption And Decreasing Absolute Risk Aversion In The Theory Of Job Search, Studies in the Economics of Search, 123, pp.109-131.

D.D. Davis et C.A. Holt [1993] : Experimental economics. Princeton University Press.

L. Denant-Boëmont et O. L'Haridon [2013]: La rationalité à l'épreuve de l'économie comportementale, Revue Française d'Economie, XXVIII, pp. 35-89.

T. Dohmen, A. Falk, D. Huffman, U. Sunde, J. Schupp, et G.G. Wagner [2011] : Individual Risk Attitudes: Measurement, Determinants, And Behavioral Consequences, Journal of the European Economic Association 9, n`3, pp.522-550.

D. Ellsberg [1961] : Risk, Ambiguity, And The Savage Axioms, The Quarterly Journal of Economics pp.643-669.

T. Eriksson, A. Poulsen et M.C. Villeval [2009], Feedback and incentives: Experimental evidence, Labour Economics, 16, n², pp. 679-688.

A. Falk et E. Fehr [2003] : Why Labour Market Experiments?, Labour Economics 10, n4, pp.399-406.

A. Falk et J. Heckman [2009] : Lab Experiments Are A Major Source Of Knowledge In The Social Sciences, Science, 326, n5952, pp. 535-538.

A. Falk et A. Ichino [2006] : Clean Evidence On Peer Effects, Journal of Labor Economics 24, n¹, pp.39-57.

C. Fershtman et U. Gneezy [2001] : Discrimination In A Segmented Society: An Experimental Approach, The Quarterly Journal of Economics 116, n¹, pp.351-377.

F. Fontaine et F. Malherbet [2013] : Accompagner les demandeurs d'emploi, Presses de Sciences Po. 
G.R. Fréchette [2009] : Laboratory Experiments: Professionals Versus Students, The Methods of Modern Experimental Economics. Oxford University Press.

M. Friedman et L.J. Savage [1948] : The Utility Analysis Of Choices Involving Risk, The Journal of Political Economy 56, n4, pp.279-304.

D. Gale et L.S. Shapley [1962] : College Admissions And The Stability Of Marriage, The American Mathematical Monthly 69, ${ }^{\circ} 1$, pp.9-15.

U. Gneezy et A. Rustichini [2000] : Pay Enough Or Don't Pay At All, The Quarterly Journal of Economics 115, $\mathrm{n}^{\circ} 3$, pp.791-810.

R. Gronau [1971] : Information And Frictional Unemployment, The American Economic Review 61, n³, pp.290-301.

G.W. Harrison, R.M. Harstad, et E.E. Rutström [2004] : Experimental Methods And Elicitation Of Values, Experimental Economics 7, n², pp.123-140.

G.W. Harrison et P. Morgan [1990] : Search Intensity In Experiments, The Economic Journal $100, n^{\circ} 401$, pp.478-486.

R. Hertwig, G. Barron, E.U. Weber, et I. Erev [2004] : Decisions From Experience And The Effect Of Rare Events In Risky Choice, Psychological Science 15, n`8, pp.534-539.

J.D. Hey [1987] : Still Searching, Journal of Economic Behavior \& Organization 8, n¹, pp.137-144.

J.D. Hey [1982] : Search For Rules For Search, Journal of Economic Behavior \& Organization $3, n^{\circ} 1$, pp.65-81.

J.D. Hey [1981] : Are Optimal Search Rules Reasonable? And Vice Versa?(And Does It Matter Anyway?), Journal of Economic Behavior \& Organization 2, ${ }^{\circ}{ }^{\circ}$, pp.47-70.

E. Hoffman, K. McCabe, K. Shachat, et V. Smith [1994] : Preferences, Property Rights, And Anonymity In Bargaining Games, Games and Economic Behavior 7, n`3, pp.346-380.

J.H. Kagel et A.E. Roth [2000] : The Dynamics Of Reorganization In Matching Markets: A Laboratory Experiment Motivated By A Natural Experiment, The Quarterly Journal of Economics 115, $\mathrm{n}^{\circ} 1$, pp.201-235.

D. Kahneman et A. Tversky [1979] : Prospect Theory: An Analysis Of Decision Under Risk, Econometrica, 47, $\mathrm{n}^{\circ} 2$, pp.263-291.

A.S. Kelso Jr et V.P. Crawford [1982] : Job Matching, Coalition Formation, And Gross Substitutes, Econometrica, 50, n6, pp.1483-1504.

J.O. Ledyard [1994] : Public goods: A survey of experimental research. Document de travail.

S.D. Levitt et J.A. List [2009] : Field Experiments In Economics: The Past, The Present, And The Future, European Economic Review 53, n¹, pp.1-18.

S.D. Levitt et J.A. List [2007] : What Do Laboratory Experiments Measuring Social Preferences Reveal About The Real World?, Journal of Economic Perspectives 21, $\mathrm{n}^{\circ} 2$, pp.pp. 153-174.

S.A. Lippman et J. McCall [1976] : The Economics Of Job Search: A Survey, Economic Inquiry $14, \mathrm{n}^{\circ} 2$, pp.155-189.

J.A. List [2006] : Field Experiments: A Bridge Between Lab And Naturally Occurring Data,

B.E. Journal of Economic Analysis \& Policy 6, $n^{\circ} 2$, Article 8. 
J.A. List [2004] : The Nature And Extent Of Discrimination In The Marketplace: Evidence From The Field, Quarterly Journal of Economics 119, n¹, pp.49-89.

J.A. List [2003] : Does Market Experience Eliminate Market Anomalies?, Quarterly Journal of Economics 118, $n^{\circ} 1$, pp.41-72.

J.A. List et I. Rasul [2011] : Field Experiments In Labor Economics, Handbook of labor economics 4, pp.103-228.

M.J. Machina [2009] : Risk, Ambiguity, And The Rank-dependence Axioms, The American Economic Review pp.385-392.

D.T. Mortensen et C.A. Pissarides [1999] : New Developments In Models Of Search In The Labor Market, Handbook of labor economics 3, pp.2567-2627.

M. Niederle, D.D. Proctor, et A.E. Roth [2006] : What Will Be Needed For The New Gastroenterology Fellowship Match To Succeed?, Gastroenterology 130, n¹, pp.218-224.

M. Niederle et L. Vesterlund [2007] : Do Women Shy Away From Competition? Do Men Compete Too Much?, The Quarterly Journal of Economics 122, n³, pp.1067-1101.

S. Rendon [2006] : Job Search And Asset Accumulation Under Borrowing Constraints*, International Economic Review 47, $n^{\circ} 1$, pp.233-263.

A.E. Roth [2010] : Is Experimental Economics Living Up To Its Promise?, The methods of modern experimental economics, Oxford University Press.

A.E. Roth [2008] : What Have We Learned From Market Design?*, The Economic Journal 118, n527, pp.285-310.

A.E. Roth [1988] : Laboratory Experimentation In Economics: A Methodological Overview, The Economic Journal 98, n`393, pp.974-1031.

A.E. Roth et J.H. Kagel [1995] : The handbook of experimental economics. Princeton University Press.

A.E. Roth et X. Xing [1994] : Jumping The Gun: Imperfections And Institutions Related To The Timing Of Market Transactions, The American Economic Review pp.992-1044.

A. Rubinstein [1982] : Perfect Equilibrium In A Bargaining Model, Econometrica: Journal of the Econometric Society pp.97-109.

L. Samuelson [2005] : Economic Theory And Experimental Economics, Journal of Economic Literature 43, n¹, pp.65-107.

A. Schotter et Y.M. Braunstein [1981] : Economic Search: An Experimental Study, Economic Inquiry $19, \mathrm{n}^{\circ} 1$, pp.1-25.

L.S. Shapley et M. Shubik [1971] : The Assignment Game I: The Core, International Journal of Game Theory 1, $\mathrm{n}^{\circ} 1, \mathrm{pp} .111-130$.

V.L. Smith [1982] : Microeconomic Systems As An Experimental Science, American Economic Review 72, n5, pp.923-955.

V.L. Smith [1962] : An Experimental Study Of Competitive Market Behavior, The Journal of Political Economy 70, n², pp.111-137.

J. Sonnemans [1998] : Strategies Of Search, Journal of economic behavior \& organization $35, \mathrm{n}^{\circ} 3$, pp.309-332.

P.P. Wakker [2010] : Prospect theory: For risk and ambiguity. Cambridge University Press. 